\title{
SUR LES ÉQUATIONS DIFFÉRENTIELLES LINÉAIRES
}

\section{A COEFFICIENTS ALGÉBRIQUES}

\author{
PAR \\ C. GUICHARD \\ a RENNES.
}

Soit:

$$
\frac{d^{n} z}{d x^{n}}+R_{1} \frac{d^{n-1} z}{d x^{n-1}}+\cdots+R_{n} z=0
$$

une équation différentielle linéaire et homogène, oì les $R_{i}$ sont des fonctions rationnelles de deux variables $x, y$ liées par une équation algébrique:

$$
F(x, y)=0 \text {. }
$$

Soit $x=a, y=b$, un point de ramification d'ordre $m$ de la courbe (2). Supposons que pour $x=a, y=b, R_{1}, R_{2}, \ldots, R_{n}$ restent finis. .Je vais démontrer le théorème suivant:

Si la variable $x$ tourne $m$ fois autour du point a, les $n$ intégrales de l'équation (I) reprennent leur valeur initiale.

Je démontrerai ce théorème en supposant $n=4$. On verra facile. ment que la démonstration s'étend au cas général. J'écris l'équation ( $\mathrm{r}$ ) sous la forme suivante:

$$
\begin{gathered}
(x-a)^{4} \frac{d^{4} z}{d x^{4}}+(x-a)^{3} \cdot P_{1} \cdot \frac{d^{3} z}{d x^{3}}+(x-a)^{2} P_{2} \cdot \frac{d^{2} z}{d x^{2}} \\
+(x-a) P_{3} \cdot \frac{d z}{d x}+P_{4}=0 .
\end{gathered}
$$


C. Guichard.

En vertu de l'hypothèse faite sur les coefficients, $P_{1}, P_{2}, P_{3}, P_{4}$ contiennent respectivement en facteur, $x-a,(x-a)^{2},(x-a)^{3},(x-a)^{4}$.

Cela posé, je ramène les $m$ portions de plan qui se raccordent au point a à la forme élémentaire par le changement de variable:

$$
x-\imath=\xi^{n} .
$$

L'équation (3) se transforme alors en la suivante:

$$
\xi^{4} \frac{l^{4} z}{d \xi^{4}}+Q_{1} \xi^{l^{2}} l^{l^{3} z}+Q_{2} \xi^{2} \frac{d^{2} z}{d \xi^{2}}+Q_{3} \xi \frac{d z}{d \xi}+Q_{4} z=0 .
$$

Pour calculer les coefficients Q je remarque que l'on a:

$$
(x-1) \cdot \frac{d F}{d x}=\frac{\mathrm{I}}{m} \cdot \xi \frac{d F}{d \xi} .
$$

En prenant pour $F$ successivement, $z,(x-a) \frac{d z}{d x}, \ldots$ on aura:

$$
\begin{gathered}
(x-a) \frac{d z}{d x}=\frac{1}{m} \cdot \xi \frac{d z}{d \xi}, \\
\left.(x-a) \frac{d}{d x}\left|(x-a) \frac{d z}{d x}\right|=\frac{1}{m^{2}} \cdot \xi \cdot \frac{d}{d \xi} \mid \xi \frac{d z}{d \xi}\right]
\end{gathered}
$$

d'oin:

$$
(x-a)^{2} \frac{d l^{2} z}{d x^{2}}=\frac{1}{m n^{2}}\left|\xi^{2} \frac{d^{2} z}{d \xi^{2}}-(m-1) \xi \frac{d z}{d \xi}\right|
$$

Je dis que d'une manière générale on a:

$$
(x-a)^{q} \frac{l^{l} z}{d x^{q}}=\frac{\mathrm{I}}{m n^{q}}\left[\xi^{q} \frac{d^{q} z}{d \vec{\xi}^{\prime l}}+a_{l_{1}} \xi^{\eta-1} \frac{d^{q-1} z}{d \xi^{1-1}}+a_{q^{2}} \xi^{q-2} \frac{d^{q-2} z}{d \xi^{q-2}}+\ldots\right] .
$$

Il suffit pour cela de démontrer que si cette formule est exacte pour une valeur de $q$, elle l'est encore pour la valeur suivante.

Appliquons la formule (5) aux deux membres de la relation (6). On aura:

$$
\begin{gathered}
(x-a)^{q+1} \frac{d^{q+1} z}{d x^{q+1}}+q(x-a)^{q} \frac{d^{q} z}{d x^{q}}=\frac{\mathrm{I}}{m^{q+1}}\left[\xi^{\prime+1} \frac{d^{q+1} z}{d \xi^{\prime q+1}}+a_{q 1} \xi^{\prime} \frac{d^{q q} z}{d \xi^{q}}+\ldots\right] \\
+\frac{\mathrm{I}}{m^{q+1}}\left[q \cdot \xi^{q} \frac{d q^{q} z}{d \xi^{q}}+(q-\mathrm{I}) a_{q 1} \xi^{\xi^{-1}} \frac{d^{q-1} z}{d \xi^{q}-1}+\ldots\right]
\end{gathered}
$$


Sur les équations différentielles linéaires à coefficients algébriques.

Cette dernière formule établit l'exactitude de la propriété énoncée. Elle donne pour le calcul des coefficients a les formules de récurrence:

$$
\begin{aligned}
& a_{q+1,1}=a_{q, 1}-(m-1) q, \\
& a_{q+1,2}=a_{q, 2}+[-m q+q-1] a_{q, 1},
\end{aligned}
$$

Je n'insiste pas davantage sur ce calcul, car il n'est pas nécessaire pour arriver à notre but de connaitre la valeur de ces coefficients.

lin portant les valeurs de $(x-a)^{q} \frac{d^{q} z}{d x^{q}}$ dans l'équation (3) on aura:

$$
\begin{aligned}
& Q_{1}=a_{41}+m P_{1}, \\
& Q_{2}=a_{42}+a_{31} m P_{1}+m^{2} I_{2}^{\prime}, \\
& Q_{3}=a_{43}+a_{32} m P_{1}+a_{21} m^{2} P_{2}+m^{3} P_{3} \\
& Q_{4}=m^{4} P_{4} .
\end{aligned}
$$

$P_{1}, P_{2}, P_{3}, P_{4}$ deviennent des fonctions de $\xi$ qui contiennent respectivement on facteur, $\xi^{m}, \xi^{2 m}, \xi^{3 m}, \xi^{4 m}$.

Les fonctions $Q_{1}, Q_{2}, Q_{3}, Q_{4}$ sont des fonctions holomorphes de $\xi$ dans le voisinage de $\boldsymbol{\xi}=$ o. Nous poserons:

$$
Q_{i}=\sum_{0}^{\infty} A_{i}^{i} \xi^{\prime \prime}
$$

Forınons maintenant la fonction caractéristique de l'équation (4), qu'on obtient, comme on le sait, en remplaçant dans le premier membre de cette équation $z$ par $\xi^{r}$. Nous obtenons:

$$
\xi^{r} F(r)+\xi^{r+1} \varphi_{1}(r)+\xi^{r+2} \varphi_{2}(r)+\ldots
$$

où:

$$
\begin{gathered}
F(r)=r(r-\mathrm{I})(r-\mathrm{I})(r-3)+A_{0}^{1} r(r-\mathrm{I})(r-2) \\
+A_{0}^{2} r(r-\mathrm{I})+A_{0}^{3} r+A_{0}^{4}, \\
\varphi_{k}(r)=A_{k}^{1} r(r-\mathrm{I})(r-2)+A_{k}^{2} r(r-\mathrm{I})+A_{k}^{3} r+A_{k}^{4} .
\end{gathered}
$$

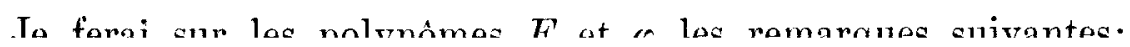


Remarque I. Les coefficients $A_{0}^{1}, A_{0}^{2}, A_{0}^{3}, A_{0}^{4}$ ont respectivement pour valeur: $a_{41}, a_{42}, a_{43}$, o; ils ne dépendent pas des coefficients des fonctions $P$. Il en résulte qu'on ne change pas la valeur de $F(r)$ si l'on suppose que dans l'équation (3) on remplace $P_{1}, P_{2}, P_{3}, P_{4}$ respectivement par $x-a,(x-a)^{2},(x-a)^{3},(x-a)^{4}$. Dans ces conditions, l'équation caractéristique de (3) aurait pour racines $0,1,2,3$. Dans l'équation transforméc (4) les racines de l'équation caractéristique seront alors $\circ, m, 2 m, 3 m$. Ce sont aussi les racines de $F(r)$. D’une manière générale, l'équation:

$$
\begin{gathered}
r(r-1) \ldots(r-q+1)+a_{q 1} r(r-1) \ldots(r-q+2) \\
+a_{q 2} r(r-1) \ldots(r-q+3)+\ldots=0
\end{gathered}
$$

adinet pour racines:

$$
0, m, 2 m, \ldots,(q-1) m
$$

ce qui donnerait une nouvelle méthode pour calculer les coefficients $a$.

Remarque 2. Les polynômes $\varphi_{1}, \varphi_{2}, \ldots, \varphi_{m-1}$ sont identiquement nuls. En effet les fonctions $Q$ ne renferment pas de termes dont le degré est $1,2, \ldots, m-1$.

Remarque 3. Les polynòmes $\varphi_{m}, \varphi_{m+1}, \ldots, \varphi_{2 m-1}$ ont pour racines communes:

$$
0, m, 2 m \text {. }
$$

En effet si $k_{i}$ est le coefficient de $\xi^{n+i}$ dans $P_{1}$ on a:

$$
\varphi_{m+i}=m \cdot k_{i}\left[r(r-1)(r-2)+a_{31} r(r-1)+a_{32} r\right] .
$$

Remarque 4. On voit de mêmo:

$1^{\mathrm{e}}$ que les polynòmes $\varphi_{2 n}, \varphi_{2 m+1}, \ldots, \varphi_{3 m-1}$ ont pour racines communes

$$
u, n
$$

$2^{e}$ que les polynómes $\varphi_{3 m}, \varphi_{3 m+1}, \ldots, \varphi_{4 m-1}$ ont pour racine commune

o. 
Sur les équations différenticlles linéaires à coefficients algébriques.

Cela posé, cherchons à intégrer. l'équation (4) en prenant pour $z$ une série de la forme:

$$
z=\sum_{0}^{\infty} c_{i} \xi^{i}
$$

On aura pour déterminer $c_{i}$ l'équation:

$$
c_{i} F(\imath)+c_{i-1} \varphi_{1}(i-1)+c_{i-2} \varphi_{2}(i-2)+\ldots+c_{0} \varphi_{i}(0) \cdots 0 .
$$

Donnons-nous arbitrairement $c_{0}$. L'équation (8) donne pour $c_{1}, c_{2}, \ldots, c_{m-1}$ la valeur $\circ$. L'équation qui détermine $c_{m}$ :

$$
c_{m} F(m)+c_{m-1} \dot{\varphi}_{1}(m-1)+\ldots+c_{0} \varphi_{m}(0)=0
$$

se réduit à une identité. On pourra prendre $c_{m}$ arbitrairement. On aura ensuite o pour valeur de $c_{m+1}, \ldots, c_{2 m-1}$. Puis l'équation qui détermine $c_{2 n}$, se réduit à une identité; $c_{2 m}$ pourra être pris arbitrairement. Ensuite on trouvera o pour les coefficients $c_{2 m+1}, c_{2 m+2}, \ldots, c_{3 m-1}$. On pourra prendre arbitrairement $c_{3 m}$. A partir de là les équations ne deviendront jamais identiques. Les coefficients $c_{3 m+1}, \ldots, c_{4 m-1}$ seront encore nuls. Mais après le terme $c_{4 m}$, il pourra y avoir des coefficients dont l'indice n'est pas divisible par $m$.

On démontre dans la théorie des équations différentielles que la série obtenue est convergente et donne l'intégrale générale. C'est une fonction uniforme de $\xi$ et par suite de $x, y$ près du point de ramification. 